\title{
Fingerprint Quality Indices for Predicting Authentication Performance
}

\author{
Yi Chen ${ }^{1}$, Sarat Dass ${ }^{2}$, and Anil Jain ${ }^{1}$ \\ 1 Department of Computer Science and Engineering \\ 2 Department of Statistics \\ Michigan State University, East Lansing, MI, 48823 \\ \{chenyi1, jain\}@cse.msu.edu, sdass@stt.msu.edu
}

\begin{abstract}
The performance of an automatic fingerprint authentication system relies heavily on the quality of the captured fingerprint images. In this paper, two new quality indices for fingerprint images are developed. The first index measures the energy concentration in the frequency domain as a global feature. The second index measures the spatial coherence in local regions. We present a novel framework for evaluating and comparing quality indices in terms of their capability of predicting the system performance at three different stages, namely, image enhancement, feature extraction and matching. Experimental results on the IBM-HURSLEY and FVC2002 DB3 databases demonstrate that the global index is better than the local index in the enhancement stage (correlation of 0.70 vs. 0.50 ) and comparative in the feature extraction stage (correlation of 0.70 vs. 0.71 ). Both quality indices are effective in predicting the matching performance, and by applying a quality-based weighting scheme in the matching algorithm, the overall matching performance can be improved; a decrease of $1.94 \%$ in EER is observed on the FVC2002 DB3 database.
\end{abstract}

\section{Introduction}

Fingerprint images are usually obtained under different conditions of the skin of a finger (e.g., dry, wet, creased/wrinkled, or abraded), the ergonomics of the acquisition system (e.g., ease of use, alignment and positioning), and the inherent limitations of the sensing equipment (e.g., shadow from optical sensors and electrical noise from capacitive sensors). These conditions, in turn, affect the quality of the acquired fingerprint images (see Figures 1 (a-c)). Fingerprint quality is usually defined as a measure of the clarity of the ridge and valley structures, as well as the "extractability" of features (such as minutiae and singularity points). Poor quality fingerprint images often result in spurious and missed features, and thus severely degrade the performance of an authentication system by increasing the false reject and false accept rates. Recently, NIST [1] has shown that the performance of a fingerprint authentication system is mostly affected, among other factors, by fingerprint image quality. Therefore, it is desirable to assess the quality of a fingerprint image to improve the overall performance of a fingerprint authentication system. 
Many on-going and past efforts have tried to address the problem of assessing fingerprint image quality. Bolle et al. [2] used ratio of directional area to nondirectional area as a quality measure. Hong et al. [3] and Shen et al. [4] applied Gabor filters to identify blocks with clear ridge and valley patterns as good quality blocks. Ratha and Bolle [5] computed the ratio of energy distribution in two subjectively selected frequency bands based on the WSQ (Wavelet Scalar Quantization) compressed fingerprint images. Lim et al. [6] combined local and global spatial features to detect low quality and invalid fingerprint images. The most recent work by Tabassi et al. [1] presented a novel definition of fingerprint quality as a predictor for matching performance. They consider quality assessment as a classification problem and use the quality of extracted features to estimate the quality label of a fingerprint image. This approach is effective only when the feature extraction algorithm is reliable and is computationally efficient.

In this paper, we propose two new fingerprint quality indices. The first index measures the entropy of the energy distribution in the frequency domain. The second estimates the local coherence of gradients in non-overlapping blocks. We propose a framework for evaluating and comparing quality indices by assessing how well they predict the system performance at three processing stages: (i) image enhancement, (ii) feature extraction and (iii) matching. Our goal is to determine how each processing stage will be affected by the image quality, and to compare the two quality indices in terms of their predictive capabilities. We also adopt a quality-based weighting scheme to improve the matching performance. To the best of our knowledge, this systematic framework is novel.

The rest of the paper is organized as follows. Section 2 describes in detail the algorithms of each proposed quality index. Section 3 introduces the new framework for evaluating fingerprint quality indices. In Section 4, experimental results are provided and discussed. Summary and future work are included in Section 5 .

\section{$2 \quad$ Fingerprint Quality Indices}

\subsection{A Quality Index in the Frequency Domain}

Given a digital image of size $M \times N$, the two-dimensional Discrete Fourier Transformation (DFT) evaluated at the spatial frequency $\left(\frac{2 \pi k}{M}, \frac{2 \pi l}{N}\right)$ is given by

$$
F(k, l)=\frac{1}{N M} \sum_{i=0}^{N-1} \sum_{j=0}^{M-1} f(i, j) e^{-\iota 2 \pi\left(\frac{k i}{N}+\frac{l j}{M}\right)}, \iota=\sqrt{-1},
$$

where $f(i, j)$ refers to the gray level intensity at pixel $(i, j)$ of the image. Although DFT produces a complex-valued output, only the power spectrum $P(k, l) \equiv$ $|F(k, l)|^{2}$ is often used as it contains most of the information regarding the geometric structure of an image.

The ridge frequency in a fingerprint image is generally around 60 cycles per image width/height [8]. Since the image width/height is usually between 120 and 1000 pixels, the dominant ridge frequencies should be between $60 / 1000=0.06$ 


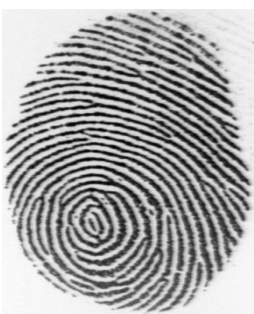

(a)

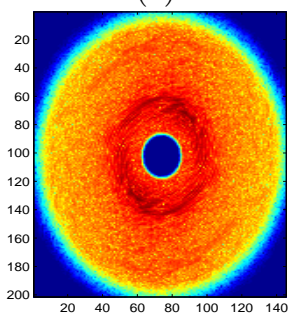

(d)

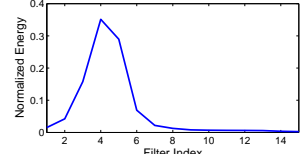

(g)

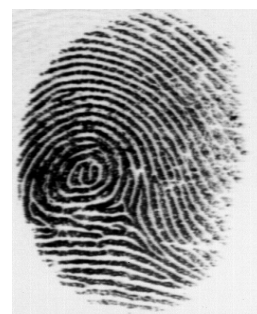

(b)

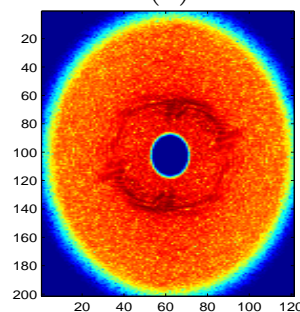

(e)

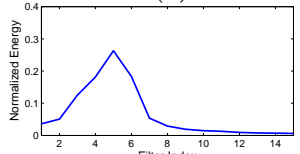

(h)

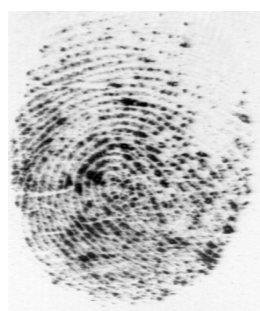

(c)

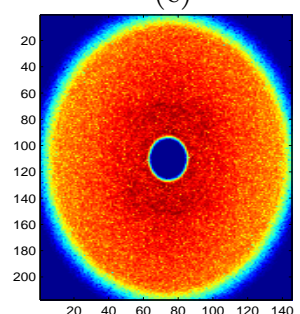

(f)

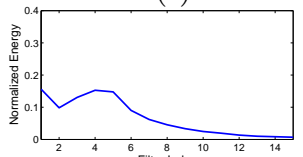

(i)

Fig. 1. Computing the quality index $Q_{f}$ from the power spectrum: Panels (a-c) show three fingerprint images in the decreasing order of quality; Panels (d-f) show their corresponding power spectrums; Panels (g-i) show the energy concentrations in the region of interest. The values of $Q_{f}$ for the three images are 1.0, 0.6, and 0.3 , respectively.

and $60 / 120=0.5$. Therefore, the region of interest $(\mathrm{ROI})$ of the power spectrum is defined to be an annular band with radius ranging from 0.06 to 0.5 . Figures $1(\mathrm{a}-\mathrm{c})$ show three fingerprint images of varying quality with their corresponding power spectrums in the ROI shown in Figures 1(d-f). Note that, the fingerprint image with good quality (Figure 1(a)) presents strong ring patterns in the power spectrum (Figure 1(d)), while a poor quality fingerprint (Figure 1(c)) presents a more diffused power spectrum (Figure 1(f)). The global quality index will be defined in terms of the energy concentration in this ROI.

We use a family of Butterworth low-pass filters to extract the ring features from the ROI. A Butterworth function [7], indexed by $m$ and $n$, is defined as

$$
H(k, l \mid m, n)=\frac{1}{1+\frac{1}{m^{2 n}}\left(\left(\frac{k-a}{M}\right)^{2}+\left(\frac{l-b}{N}\right)^{2}\right)^{n}},
$$

where $(k, l)$ is the pixel index in the power spectrum corresponding to the spatial frequency $\left(\frac{2 \pi k}{M}, \frac{2 \pi l}{N}\right)$ and $(a, b)$ is the location of the center of the power spectrum corresponding to spatial frequency $(0,0)$. The Butterworth function generates a low-pass filter with the cutoff frequency given by $m$ and the filter order given by $n$. The value of $n$ controls the steepness of the drop at the cutoff frequency; the larger the value of $n$, the closer $H$ is to an idealized step function. 

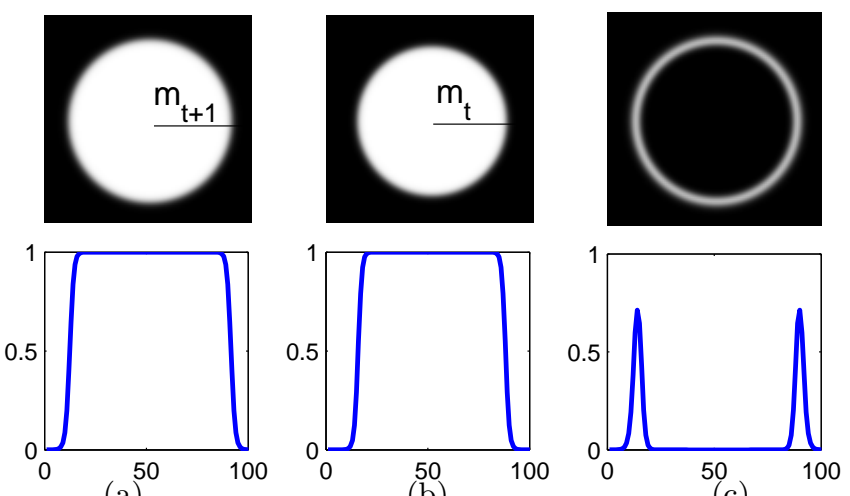

(a)

(b)

(c)

Fig. 2. Taking the differences of two consecutive low-pass filters (a) $H\left(k, l \mid m_{t+1}, n\right)$ and (b) $H\left(k, l \mid m_{t}, n\right)(n=20)$ to obtain a bandpass filter (c) $R_{t}(k, l)$.

We construct a total of $T$ equally spaced bandpass filters, $R_{t}$, by taking differences of two consecutive Butterworth functions, that is,

$$
R_{t}(k, l)=H\left(k, l \mid m_{t+1}, n\right)-H\left(k, l \mid m_{t}, n\right),
$$

where $m_{t}=0.06+t \frac{0.5-0.06}{T}$ and $t=0,1,2, \ldots,(T-1)$. The construction of $R_{t}(k, l)$ from $H\left(k, l \mid m_{t+1}, n\right)$ and $H\left(k, l \mid m_{t}, n\right)$ is shown graphically in Figure 2 . For every $t, R_{t}$ captures the energy in an annular band with frequencies from $m_{t}$ to $m_{t+1}$. The energy concentrated in the $t$-th band is computed by

$$
E_{t}=\sum_{k=0}^{N-1} \sum_{l=0}^{M-1} R_{t}(k, l) P(k, l),
$$

and the normalized energy for the $t$-th bandpass filter is defined as $P_{t}=\frac{E_{t}}{\sum_{t=0}^{T-1} E_{t}}$. In Figures 1(g-i), we plot the distribution of $P_{t}$ for $T=15$ bandpass filters. A good quality image has a more peaked energy distribution while poor ones have more diffused distribution. The extent of energy concentration is given by the entropy

$$
E=-\sum_{t=0}^{T-1} P_{t} \log P_{t}
$$

which achieves the maximum value $\log T$ when the distribution is uniform and decreases when the distribution is peaked. Our quality score is defined as

$$
Q_{f}=\log T-E
$$

so that a fingerprint image with good (bad) quality will have a higher (lower) value of $Q_{f}$. We have normalized $Q_{f}$ on the database so that the values lie between 0 and 1 .

\subsection{A Quality Index in the Spatial Domain}

To assess fingerprint image quality in a local region, we partition a given image into a lattice of blocks of size $b \times b$. An algorithm to distinguish the fingerprint 
foreground from the background is then applied as described in [2]. For each foreground block $B$, let $g_{s}=\left(g_{s}^{x}, g_{s}^{y}\right)$ denote the gradient of the gray level intensity at site $s \in B$. The covariance matrix of the gradient vectors for all $b^{2}$ sites in this block is given by

$$
J=\frac{1}{b^{2}} \sum_{s \in B} g_{s} g_{s}^{T} \equiv\left[\begin{array}{ll}
j_{11} & j_{12} \\
j_{21} & j_{22}
\end{array}\right] .
$$

The above symmetric matrix is positive semidefinite with eigenvalues

$$
\begin{aligned}
& \lambda_{1}=\frac{1}{2}\left(\operatorname{trace}(J)+\sqrt{\operatorname{trace}^{2}(J)-4 \operatorname{det}(J)}\right) \\
& \lambda_{2}=\frac{1}{2}\left(\operatorname{trace}(J)-\sqrt{\operatorname{trace}^{2}(J)-4 \operatorname{det}(J)}\right),
\end{aligned}
$$

where $\operatorname{trace}(J)=j_{11}+j_{12}, \operatorname{det}(J)=j_{11} j_{22}-j_{12}^{2}$ and $\lambda_{1} \geq \lambda_{2}$. The normalized coherence measure is defined as

$$
\tilde{k}=\frac{\left(\lambda_{1}-\lambda_{2}\right)^{2}}{\left(\lambda_{1}+\lambda_{2}\right)^{2}}=\frac{\left(j_{11}-j_{22}\right)^{2}+4 j_{12}^{2}}{\left(j_{11}+j_{22}\right)^{2}},
$$

with $0 \leq \tilde{k} \leq 1$. This measure reflects the clarity of the local ridge-valley orientation in each foreground block $B$. If the local region has a distinct ridge-valley orientation, then $\lambda_{1} \gg \lambda_{2}$ results in $\tilde{k} \approx 1$. On the contrary, if the local region is of poor quality, we obtain $\lambda_{1} \approx \lambda_{2}$ and consequently $\tilde{k} \approx 0$.

A single quality score can be computed as the weighted average of the blockwise coherence measures given by

$$
Q_{s}=\frac{1}{r} \sum_{i=1}^{r} w_{i} \tilde{k}_{i}
$$

where $r$ is the total number of foreground blocks, and the relative weight $\omega_{i}$ for the $i$-th block centered at $l_{i}=\left(x_{i}, y_{i}\right)$ is determined by

$$
\omega_{i}=\exp \left\{-\left\|l_{i}-l_{c}\right\|^{2} /(2 q)\right\},
$$

where $l_{c}$ is the centroid of foreground fingerprint, and $q$ is a normalization constant, which reflects the contribution for blocks with respect to the distance from the centroid [5]. Generally, regions near the centroid of a fingerprint receive higher weights, since they are likely to provide more information than the peripheral.

Figure 3 shows the local quality maps of the three fingerprint images and their overall quality indices. We have also normalized $Q_{s}$ on the database so that the values lie between 0 and 1 .

\section{Evaluation Criteria}

In this section, an evaluation criteria is developed for assessing the performance of image enhancement, feature extraction and matching with respect to the proposed quality indices. 


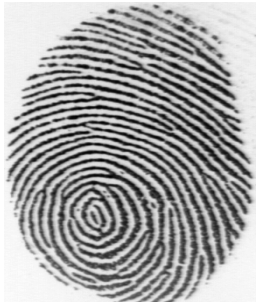

(a)

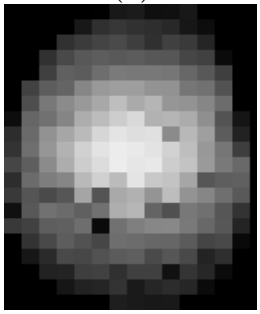

(d)

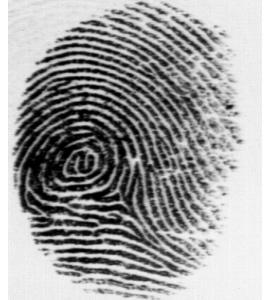

(b)

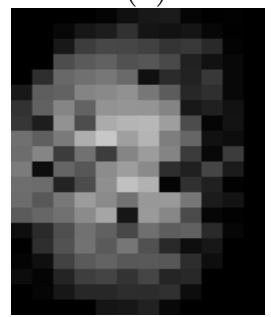

(e)

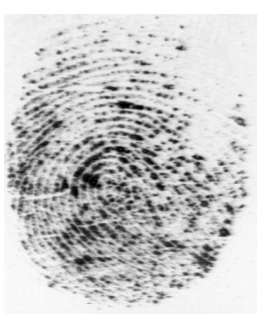

(c)

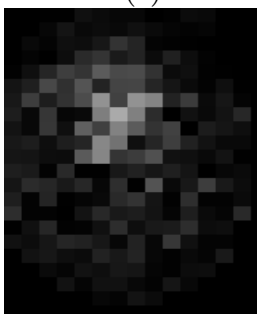

(f)

Fig. 3. Computing the quality index $Q_{s}$ using the spatial coherence measure. Panels (a-c) are the fingerprint images. Panels (d-f) are the block-wise values of $\tilde{k}$; blocks with brighter color indicate higher quality in the region. The values of $Q_{s}$ for the three fingerprint images are $0.95,0.56$, and 0.20 , respectively.

\subsection{Predicting the Image Enhancement Performance}

Our goal is to first quantify the robustness of enhancement for varying values of $Q_{f}$ and $Q_{s}$. A fingerprint image with high values of $Q_{f}$ and $Q_{s}$ should be less sensitive (or more robust) to the tuning parameters of an enhancement algorithm than those with low $Q_{f}$ and $Q_{s}$ values. The following method is developed to quantify this sensitivity with regard to the tuning of an enhancement algorithm.

Given an enhancement algorithm $E$, we tune the parameters to obtain a modified version called $E^{\prime}$. Run $E$ and $E^{\prime}$ separately on a fingerprint image to generate two enhanced images $I$ and $I^{\prime}$. Let $A=\left(g^{1}, g^{2}, \ldots, g^{u}\right)$ and $B=$ $\left(h^{1}, h^{2}, \ldots, h^{v}\right)$ be the sets of minutiae detected, respectively, from $I$ and $I^{\prime}$. Compute $p$ as the number of paired minutiae in $A$ and $B$ : minutiae $g^{i}(i=$ $1, \ldots, u)$ and $h^{j}(j=1, \ldots, v)$ are said to be paired if their distances in position and orientation are within a tolerance bound of 8 pixels and 30 degrees, respectively. The robustness index $(R I)$ of a fingerprint image is given by

$$
R I=\frac{p}{u+v-p}
$$

where $(u+v-p)$ represents the total number of minutiae detected in both enhanced images. A low $R I$ value indicates large variance in the number of minutiae detected and hence poor image quality due to its sensitivity to the turning of parameters. On the contrary, high $R I$ value indicates consistency in minutiae extraction and consequently, good image quality due to its robustness to parameter tuning (Figure 4). 


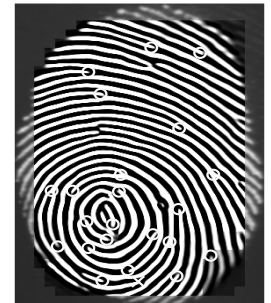

(a)

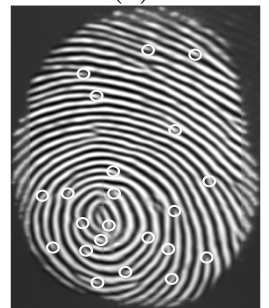

(d)

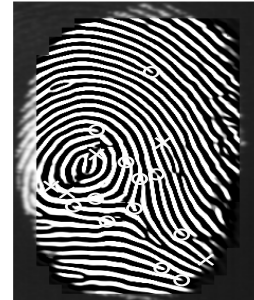

(b)

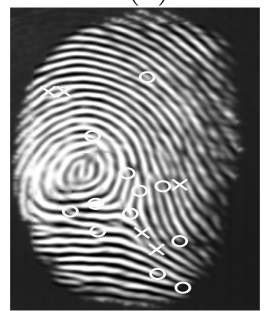

(e)

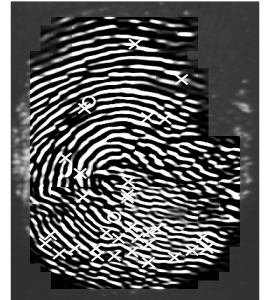

(c)

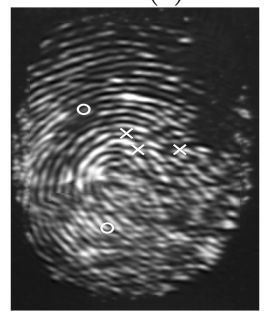

(f)

Fig. 4. Sensitivity to the tuning parameters of an enhancement algorithm. Input images are those shown in Figures 3(a-c). Panels (a-c) are obtained using $E$ while panels (df) are obtained using $E^{\prime}$. Minutiae consistently extracted from both algorithms are considered robust (o), whereas minutiae detected only by $E$ or $E^{\prime}$ are non-robust $(\times)$.

\subsection{Predicting the Feature Extraction Performance}

The effects of image quality with regard to feature extraction performance can be measured using the goodness index (GI) defined as

$$
G I=\frac{p}{t}-\frac{a+b}{u}
$$

where $p, a, b$, respectively, represent the total number of paired, missed and spurious minutiae among the $u$ detected minutiae when compared to the number of ground truth minutiae $t$ in the given fingerprint image. Here, missed minutiae refers to a ground truth minutiae that is missed by the feature extraction whereas spurious minutiae represents an extracted minutiae that is not matched with a ground truth minutiae. A low $G I$ value is obtained when the number of missed or spurious minutiae is much larger than the paired minutiae, indicating poor image quality. A high $G I$ value, on the contrary, indicates good quality as most minutiae are correctly matched.

\subsection{Predicting the Matching Performance}

When matching scores are available, a Receiver Operating Characteristic (ROC) curve is plotted to reflect the performance of the matching algorithm. One effective evaluation criterion for a quality index is to rank the ROC as a function of image quality. More specifically, we can divide the quality scores into $r$ equally numbered bins (from low to high) and plot $r$ ROC curves, with the $i$-th curve reflecting the matching performance after images in the first $i, 0 \leq i \leq 4$ bins are pruned. The 0 -th bin is by convention, the original database with no images 
removed. If a quality index is a good predictor of the matching performance, the ROC curves should consistently rise as more poor quality images are excluded.

\subsection{Incorporating Local Quality into the Matching Algorithm}

We propose to incorporate the local coherence measure, $\tilde{k}_{i}$, into the fingerprint matching algorithm [12] that accounts for the reliability of the extracted minutiae points. Prior to finding the matching score between a pair of fingerprint images, we need to align them to remove effects of any translation and rotation of the finger. This is done by maxmizing

$$
W=\sum_{i=1}^{p} \sqrt{\tilde{k}_{f(i)}^{A} \times \tilde{k}_{g(i)}^{B}},
$$

where $p$ is the total number of paired minutiae between $A$ and $B, \tilde{k}_{f(i)}^{A}$ and $\tilde{k}_{g(i)}^{B}$ are the local coherence measures associated with the $i$-th paired minutiae in $A$ and $B$, respectively; functions $f$ and $g$ return index of the block that contains the paired minutiae belonging to $A$ and $B$, respectively. Once $W$ is maximized, its corresponding transformation parameters are applied to align the orientation field of the pair, with both results determining the final matching score. Therefore, if the quality is high for both minutiae in a pair, this pairing will contribute more to the estimation of transformation parameters as well as the matching score than a pairing of low quality minutiae.

\section{Experimental Results}

The quality indices are tested using two databases, namely the IBM-HURSLEY database and FVC2002 DB3 [11]. The IBM-HURSLEY database contains multiple impressions of 269 fingers (a total of 900 images) taken at significantly different times, resulting in large variability in fingerprint quality. The images have different sizes but the same resolution of $500 \mathrm{dpi}$, with "true" minutiae marked by a human expert. The FVC2002 DB3 contains 800 images from 100 fingers ( 8 impressions per finger), all with the same size $(300 \times 300)$ and the same resolution (500 dpi). This database is the most difficult among the four databases in FVC2002 in terms of image quality [11]. No ground-truth is provided for this database, and hence, the quality indices for this database are tested at the matching stage, while the quality indices for IBM-HURSLEY are tested at the enhancement and the feature extraction stages.

To evaluate the enhancement performance with regard to the proposed quality indices, we employed the enhancement algorithm proposed in [9], and the minutiae feature extraction algorithm given in [10]. We apply a new combination of three tuning parameters of the enhancement algorithm, namely, the minimum inter-ridge distance, the window width and height. The default combination was $E=[12,11,11]$ and the new one is $E^{\prime}=[7,11,7]$. The $R I$ value for each fingerprint is obtained as in equation (12) and the quality indices $Q_{f}$ and $Q_{s}$ are obtained as in Section 2. Figures $5(\mathrm{a}-\mathrm{b})$ show the scatter plots of $R I$ 
versus $Q_{f}$ and $R I$ versus $Q_{s}$ on the IBM-HURSLEY database. We also sort the images in the increasing order of quality and divide them into $r=5$ bins (180 images per bin). The median and the lower and upper quartiles of the quality indices in each bin are calculated and shown in the box plots in Figures 5 (cd). It is demonstrated that $Q_{f}$ has a stronger predictive capability for $R I$, as it acquires a larger Pearson's correlation (0.70) than $Q_{s}(0.50)$. In a similar

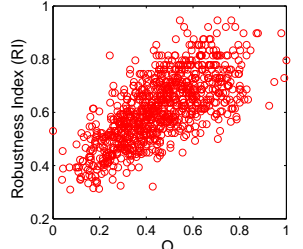

(a)

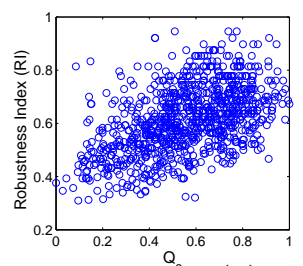

(b)

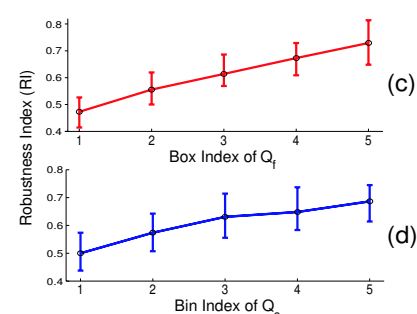

(d)

Fig. 5. The effect of the proposed quality indices on image enhancement. (a) gives the scatter plot and (c) the box plot of $R I$ versus $Q_{f}$, and (b) gives the scatter plot and (d) the box plot of $R I$ versus $Q_{s}$.

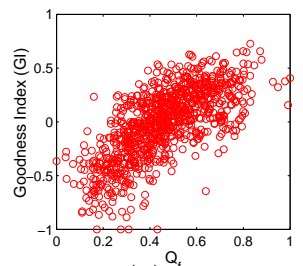

(a)

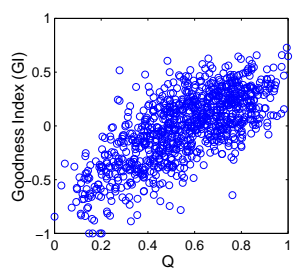

(b)

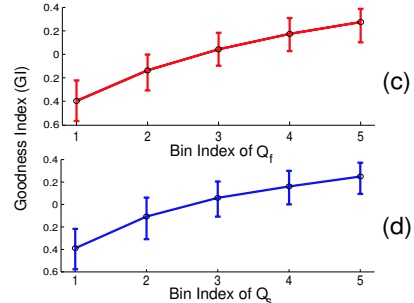

(d)

Fig. 6. The effect of the proposed quality indices on feature extraction. (a) gives the scatter plot and (c) the box plot of $G I$ versus $Q_{f}$, and (b) gives the scatter plot and (d) the box plot of $G I$ versus $Q_{s}$.

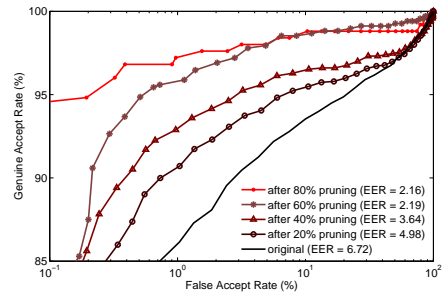

(a)

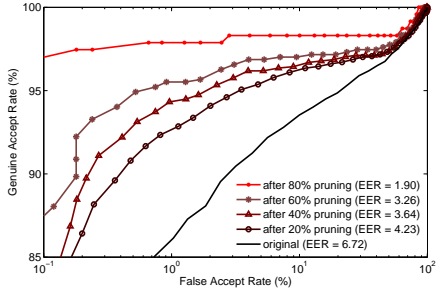

(b)

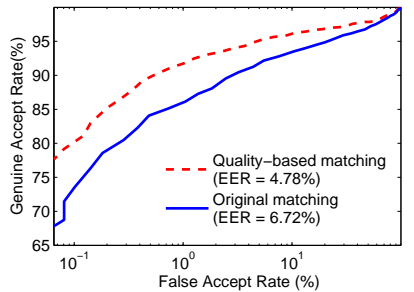

(c)

Fig. 7. Improving the matching performance by (a) pruning poor quality images with regard to $Q_{f}$ and (b) $Q_{s}$, and (c) adopting a quality-based weighting scheme in the matcher.

manner, the performance of the feature extraction algorithm (measured by $G I$ in equation (13)) is evaluated with respect to $Q_{f}$ and $Q_{s}$. Here, default settings of the enhancement algorithm is used. Figure 6 gives the corresponding results. 
Both $Q_{f}$ and $Q_{s}$ are effective in predicting the feature extraction performance with $Q_{s}$ achieving a slightly higher correlation than $Q_{f}(0.71$ vs. 0.70$)$.

Finally, a matcher proposed in [12] is adopted for evaluating and improving the matching performance with respect to the quality indices on FVC2002 DB3. Five ROC curves are plotted in Figures 7(a-b) as suggested in Section 3.3. Figure $7(\mathrm{c})$ shows the overall improvement in the matching performance when local coherence measures are incorporated by a quality-based weighting scheme in the matcher (see Section 3.4).

\section{Conclusion and Future Work}

This paper proposes two quality indices, global $\left(Q_{f}\right)$ and local $\left(Q_{s}\right)$, for fingerprint images. We compare the two in a generic evaluation framework and observe the following: (1) $Q_{f}$ has better predictive capabilities at the image enhancement stage than $Q_{s}$. This is because the image enhancement algorithm we use is based on Gabor filtering in the frequency domain, and is therefore directly related to $Q_{f}$. (2) $Q_{s}$ is slightly more effective than $Q_{f}$ at the feature extraction stage. This is because feature extraction concentrates on local details which is measured directly by $Q_{s}$. (3) Both $Q_{f}$ and $Q_{s}$ are effective in predicting and improving the matching performance. Future work includes expanding the current framework to other possible representation of fingerprints and biometric identifiers.

\section{References}

1. Tabassi, E., Wilson, C., Watson, C.: Fingerprint Image Quality. NIST research report NISTIR7151 (August, 2004).

2. Bolle, R. et al.: System and methods for determing the quality of fingerprint images. United Sates patent number US596356 (1999).

3. Hong, L., Wan, Y., Jain, A.: Fingerprint image enhancement: algorithms and performance evaluation. IEEE Transactions on Pattern Analysis and Machine Intelligence, 20 (1998) 777-789.

4. Shen, L., Kot, A., Koo, W.: Quality measures of fingerprint images. Audio- and Video-based Biometric Person Authentication, (2001).

5. Ratha, N., Bolle, R.: Fingerprint image quality estimation. IBM computer science research report $\mathrm{RC} 21622$ (1999).

6. Lim, E., Jiang, X., Yau, W.: Fingerprint quality and validity analysis. IEEE International Conference on Image Processing, 1 (2002) 469-472.

7. Rosenfeld, A., Kak, A.: Digital Picture Processing. Academic Press, (1982).

8. Hong, L., Jain, A., Pankanti, S., Bolle, R.: Fingerprint Enhancement. IEEE Workshop on Applications of Computer Vision, (1996) 202-207.

9. Hong, L., Wan. Y, Jain, A.: Fingerprint Image Enhancement: Algorithms and Performance Evaluation. IEEE Transactions on PAMI, 20-8 (1998) 777-789.

10. Jain, A., Hong, L., Bolle, R.: On-line fingerprint verification. IEEE Trasactions on Pattern Analysis and Machine Intelligence, 19-4 (1997) 302-314.

11. Maltoni, D., Cappelli, R., Wayman, J., Jain, A.: FVC2002: Second Fingerprint Verification Competition. International Conference on Pattern Recognition, 3 (2002) 811-814.

12. Jain, A., Prabhakar, S., Chen, S.: Combining Multiple Matchers for a High Security Fingerprint Verification System. Pattern Recognition Letters, 20 (1999) 1371-1379. 